\title{
Propuesta de modelo para el emprendimiento de base universitaria a través del estado del arte
}

\section{Proposal of a model for university-based entrepreneurship through the state of art}

\author{
Ana Patricia Benavides Díaz ${ }^{1^{*}}$, Dalys Guevara ${ }^{2}$ \\ ${ }^{1}$ Licenciatura en Ingeniería Mecánica Industrial, Campus Víctor Levi Sasso, Universidad Tecnológica de Panamá \\ ${ }^{2}$ Facultad de Ingeniería Industrial, Campus Víctor Levi Sasso, Universidad Tecnológica de Panamá
}

\begin{abstract}
Resumen En la actualidad, las naciones a nivel mundial se encuentran afectadas por una creciente recesión económica que trae desempleo y desmejoramiento de la calidad de vida de las poblaciones. Como respuesta a estos problemas, los economistas proponen el emprendimiento como una alternativa altamente beneficiosa; el cual no solamente amplía la cantidad de plazas de trabajo, sino que, a su vez, sirve para brindar soluciones alternas que satisfacen las necesidades de las comunidades que actualmente se encuentran cubiertas y, muchas veces, generar ideas que solucionen aquellas que no eran atendidas antes. Esta investigación tiene como objetivo elaborar un "estado del arte" sobre el emprendimiento de base universitaria, haciendo un enfoque en la relación que existe entre la educación superior y el emprendimiento. Para esto, se desenvuelven como temas centrales, el desarrollo de las habilidades emprendedoras por medio de programas y modelos de enseñanza, las motivaciones principales que llevan a los individuos a crear proyectos emprendedores y los factores que influyen en la decisión del individuo de emprender o no. Además de esto, se propone un modelo que puede ser validado en la Universidad Tecnológica de Panamá (UTP) para desarrollar una cultura emprendedora entre los estudiantes y promover la generación de ideas innovadoras, con miras a la creación de nuevas empresas.
\end{abstract}

Palabras clave Educación emprendedora, emprendimiento, emprendimiento de base universitaria, espíritu emprendedor, universidades emprendedoras.

\begin{abstract}
Nations around the world are being affected by the current economic recession that brings with it unemployment and the deterioration of the quality of life of the populations. In response to these problems, economists suggest entrepreneurship as a highly beneficial alternative, since it not only increases the number of jobs, but also serves to provide alternative solutions that satisfy the needs of the communities that are currently covered, and even generate ideas that solve those that were not addressed before. This research aims to develop a State of Art on entrepreneurship, emphasizing the relationship between higher education and entrepreneurship. For this, the development of entrepreneurial skills through programs and teaching models, the main motivations that lead individuals to develop an entrepreneurial project and the factors that influence the decision of the individual to undertake or not, are developed as central themes. In addition, a proposal of a model that can be implemented at the Universidad Tecnológica de Panamá (UTP) to develop an entrepreneurial culture among students and promote the generation of innovative ideas is presented.
\end{abstract}

Keywords Entrepreneurial education, entrepreneurship, entrepreneurial universities, entrepreneurial spirit, university-based entrepreneurship.

*Corresponding author: anapatriciabd@gmail.com

\section{Introducción}

Las naciones actuales se ven afectadas por distintos problemas en el ámbito económico y social. Uno de estos, es el incremento de la tasa de desempleo, fenómeno que afecta a la población a nivel mundial y trae consigo el aumento de la pobreza dentro de los países. De manera proporcional, el aumento del desempleo incrementa la recesión económica. A esto se le suma la falta de innovación, lo cual admite que se dé la monopolización de los mercados y se pierda la oportunidad de encontrar soluciones más óptimas y amigables, para el ambiente y los consumidores, para las necesidades que existen. El emprendimiento es considerado una herramienta muy poderosa y sirve para solucionar estos problemas que se presentan. Muchas universidades en distintos lugares del mundo han adoptado programas y métodos de enseñanza para desarrollar una cultura emprendedora en sus estudiantes, con el fin de cumplir su misión con la sociedad de mejorar la economía nacional y brindar una mejor calidad de vida para los habitantes. Existe una relación indiscutible entre la educación superior y el 
emprendimiento, ya que gracias a los conocimientos que son adquiridos en la primera, se desarrollan las ideas que permiten que se dé un emprendimiento exitoso.

Este proyecto tuvo como finalidad elaborar un "estado del arte" sobre el emprendimiento de base universitaria, con un enfoque en los factores que afectan la decisión del individuo hacia el emprendimiento, las motivaciones que lo llevan a generar un proyecto emprendedor y los modelos y programas de enseñanza utilizados para desarrollar las habilidades conceptuales, técnicas y blandas necesarias para poner en marcha una idea innovadora.

Luego de esto, se presenta una propuesta sobre el programa de emprendimiento que debe llevarse para crear una cultura emprendedora dentro de la comunidad estudiantil de la Universidad Tecnológica de Panamá.

\subsection{Objetivo}

Proponer un modelo que contribuya a generar una cultura emprendedora de base universitaria y que pueda ser aplicado en la Universidad Tecnológica de Panamá.

\section{Marco teórico}

\subsection{Evolución del concepto de emprendimiento}

El emprendimiento es una característica que ha acompañado al hombre desde el origen de la humanidad, aunque en ese momento el término no había sido definido. Esta palabra apareció por primera vez en la literatura económica gracias a Richard Cantillon, quien la definió como "el agente que compra los medios de producción a ciertos precios y los combina en forma ordenada para obtener allí un nuevo producto" [1]. Al pasar de los años, el término se ha nutrido con distintos matices, como el reconocimiento del emprendedor como un individuo que sabe tomar riesgos, identificar las oportunidades del mercado y promover la creación de innovaciones.

\subsection{Importancia del emprendimiento en la actualidad}

Al pasar de los años, el concepto de emprendimiento ha tomado más importancia debido al incremento del desempleo, la crisis de la economía de los países y la necesidad del mejoramiento de la calidad de vida de las familias. Según el Banco Mundial de Datos, hasta el año 2016, países de Latinoamérica como Chile, Colombia, Costa Rica, El Salvador, Panamá y Perú presentaron un aumento en el registro de empresas, demostrando que cada día más personas toman la decisión de abrir sus propios negocios [2]. Sin embargo, aunque el registro de nuevas empresas vaya en incremento, esto no indica que las mismas aportan una solución para los problemas de desempleo y pobreza, ya que no se asegura que las mismas resulten exitosas.

En la actualidad, los países del primer mundo se encuentran trabajando en el desarrollo de productos y servicios innovadores por medio del emprendimiento. Aquellas naciones que sean capaces de aplicar los avances tecnológicos junto con los aprendizajes y habilidades conceptuales y técnicas que se desarrollan durante la educación, podrán conseguir un incremento en su economía [3].

\subsection{El emprendimiento de base universitaria y la educación emprendedora}

Tomando en consideración lo mencionado en la sección "2.1" sobre la evolución del concepto de emprendimiento, es importante mencionar que no se ha llegado a un acuerdo sobre una definición específica, sin embargo, los autores reconocen que un emprendedor debe poseer habilidades y capacidades particulares para desarrollar un proyecto exitoso.

De allí surge la conexión entre el emprendimiento y la educación superior, siendo la segunda, la encargada de desarrollar estas habilidades en los individuos. Dada la importancia del emprendimiento en la actualidad y el compromiso de las universidades de capacitar capital humano, muchas instituciones de educación superior han desarrollado programas, estrategias de enseñanza y modelos de aprendizaje que les permiten potenciar el espíritu emprendedor de sus estudiantes. Para lograr esto, es necesario identificar cuáles son los factores que despiertan la creatividad e innovación en los individuos y, al mismo tiempo, enfocar la educación que ofrecen hacia el desarrollo de técnicas de emprendimiento.

\section{Metodología}

Para el desarrollo del "estado del arte" que se presenta en esta investigación, se utilizó como técnica la búsqueda sistemática. Esta permite la recolección detallada de información sobre un tema de interés y, al mismo tiempo, resaltar cuáles son los factores más importantes. La metodología sistemática que se empleó consta de cinco fases definidas: la identificación del campo de estudio y el periodo por analizar, la selección de las fuentes de información, la realización de la búsqueda, la gestión y depuración de resultados y, por último, el análisis de resultados [4], [5].

Para realizar un "estado del arte" de manera correcta es necesario tomar algunas consideraciones. Primero, se debe definir con exactitud el tiempo de estudio, los objetivos y el problema de estudio. Luego de esto, se deben seleccionar las fuentes que serán utilizadas para recolectar la información y asegurarse de que estas sean confiables. Posteriormente, se procede con la búsqueda de los archivos de relevancia para el estudio, los mismos deben ser identificados y seleccionados, para obtener más precisión, de manera manual. La depuración correcta de los archivos es esencial para realizar un análisis correcto del tema de investigación. Seguidamente, se procede a realizar el análisis crítico; esto tiene como objetivo la identificación de los factores relevantes en el tema de estudio y, al mismo tiempo, permite realizar una opinión sobre el tema que se está tratando [4], [5]. 


\section{Análisis de resultados}

La investigación comienza reconociendo a la educación como la base fundamental para combatir la pobreza y mejorar la calidad de vida y el compromiso que las universidades tienen de enfocar y conducir la enseñanza con miras a la investigación, la innovación y la responsabilidad social [3]. Para generar un emprendimiento de éxito es necesario que los individuos desarrollen una serie de conocimientos y habilidades específicas que les permitan desempeñar funciones y tomar decisiones. Tomando en consideración lo anterior, podemos argumentar que la educación juega un papel clave en los individuos y los prepara, por medio de los conocimientos y habilidades que son aprendidos, para solucionar problemas de manera eficiente.

Existen distintos talentos claves que deben ser desarrollados por medio de la educación para que un emprendedor sea exitoso y estos se dividen en cuatro categorías [6]. En la tabla 1 se describen estos talentos:

Tabla 1. Talentos que deben desarrollarse por medio de la educación superior para originar emprendedores de éxito [6]

\begin{tabular}{|l|l|}
\hline TALENTO & \multicolumn{1}{|c|}{ DESCRIPCIÓN } \\
\hline Conceptuales & $\begin{array}{l}\text { Permiten ver la información de una } \\
\text { manera lógica y, por consiguiente, } \\
\text { proporcionan resultados positivos en } \\
\text { cuanto a la visión que se tiene sobre una } \\
\text { empresa. }\end{array}$ \\
\hline Emprendedores & $\begin{array}{l}\text { Se desarrollan a partir de los conceptos } \\
\text { empresariales que los individuos vayan } \\
\text { adquiriendo. Estos, usualmente, son una } \\
\text { imitación de algún otro emprendedor del } \\
\text { cual se tome referencia. }\end{array}$ \\
\hline Organizativos & $\begin{array}{l}\text { Son aquellos que le permitirán a los } \\
\text { emprendedores dirigir su organización de } \\
\text { manera eficiente y eficaz para que la } \\
\text { misma logre alcanzar las metas y éxito que } \\
\text { este se propone. }\end{array}$ \\
\hline Técnicos & $\begin{array}{l}\text { Estos se desarrollan a partir de } \\
\text { conocimiento de técnicas de marketing, } \\
\text { finanzas, operaciones, recursos humanos, } \\
\text { entre otros. Son vistos como los } \\
\text { conocimientos específicos que se tendrán } \\
\text { sobre la administración de una empresa. }\end{array}$ \\
\hline
\end{tabular}

Además de esto, se debe incentivar la creatividad, la cual es considerada la facultad latente más difícil de desarrollar, ya que el cerebro está diseñado por naturaleza para reconocer y seguir patrones estables de conducta. El pensamiento lateral es aquel que permite desarrollar nuevas ideas y, es en el que hay que trabajar con mayor ahínco al momento de apuntar hacia la enseñanza emprendedora [6]. La innovación va de la mano con la creatividad; esta responde a la demanda del mercado y a sus necesidades inmediatas y, a partir de estas, se desarrollan las ideas que se van a poner en marcha por medio de nuevos emprendimientos.

Dentro de esta investigación se hará un enfoque de tres áreas específicas del emprendimiento de base universitaria: los factores influyentes en la decisión de los individuos de emprender, las motivaciones que los llevan a desarrollar un proyecto emprendedor y los programas y modelos generados por universidades e investigadores para incentivar el emprendimiento en los estudiantes. Estas áreas serán desarrolladas seguidamente.

\subsection{Factores que afectan las intenciones emprendedoras de los individuos}

Existen distintos factores que son determinantes e influyen en las intenciones de los individuos para emprender. Los autores no han llegado a un consenso sobre cómo afectan estos a los individuos, pero cada uno tiene una opinión personal sobre sus efectos. Entre los factores más discutidos se encuentran los siguientes:

- Educación emprendedora: se reconoce que la educación emprendedora que reciban los estudiantes estará ligada a las actividades emprendedoras que estos desarrollen y se encuentra en discusión si los efectos de esta son positivos o negativos. Entre los estudios que se investigaron se tienen conclusiones por parte de autores que indican que las universidades deben enfocar su educación hacia el desarrollo de la innovación y la creatividad [5]. También, se reconoce la conexión que existe entre las actitudes relacionadas con el emprendimiento y la educación enfocada en el emprendimiento, que desarrolla los perfiles necesarios para que los individuos tengan éxito en este ámbito [7]. Además de lo mencionado, muchos autores lo categorizan como un factor que tiene efectos positivos en los estudiantes [8-10], otros concluyen que no se puede definir con certeza si existe una conexión entre la educación emprendedora y el emprendimiento que se desarrolla luego de esta [11] y otros aseguran que no existe tal conexión [12]. Dentro de esta investigación, se considera la educación emprendedora como un factor que tiene un efecto positivo, argumentando que es la que ayuda al individuo a tomar la decisión de emprender o no. Gracias a la educación emprendedora, los individuos pueden identificar si poseen algún tipo de afinidad hacia el emprendimiento. De esta manera, si el individuo toma la decisión de emprender o no, existirán múltiples beneficios como son: la utilización correcta de recursos, satisfacción profesional y mejoramiento de la calidad de vida.

- Género: la desigualdad de género es un tema muy discutido en múltiples ámbitos de las sociedades humanas y en el emprendimiento no es la excepción. Los autores que argumentan sobre este factor llegan a conclusiones diversas, donde algunos opinan que el género es un factor que no influye en la decisión de emprender de los individuos [13], [14], y otros aseguran que el género masculino posee mayores intenciones emprendedoras [15]. Dentro de esta investigación se considera el género como un factor que influye en la decisión de emprender, ya que en el porcentaje de participación de las 
mujeres en el ámbito emprendedor es notablemente menor que el de los hombres. Es importante resaltar, que esta brecha porcentual ha ido disminuyendo al pasar de los años, por lo que se espera que, en un futuro, el género no sea un factor determinante.

- Personalidad: este conjunto de rasgos define el comportamiento habitual de una persona, por lo que, se considera la personalidad del individuo como un factor importante en la decisión de emprender [7], [16], [17]. Dentro de este estudio se categoriza la personalidad como un factor influyente y se reconoce que los emprendedores que se catalogan como exitosos, poseen altos puntajes en algunos rasgos de personalidad específicos como: apertura a las experiencias nuevas, responsabilidad, extraversión, amabilidad y estabilidad emocional. El éxito de estos individuos se encuentra fundamentado en su forma óptima de sacar provecho de sus habilidades blandas.

- Familiares con antecedentes emprendedores: el ser humano utiliza patrones de conducta para adquirir su manera de comportarse y, usualmente, estos son aprendidos por observación de los familiares cercanos. Los autores que fueron considerados dentro de este estudio argumentan que el poseer familiares emprendedores motiva a los individuos a seguir el camino del emprendimiento y, por consiguiente, se considera un factor que afecta de manera positiva [10], [14], [15]. Dentro de esta investigación se seguirá esa línea de pensamiento, tomando en consideración que los padres emprendedores les inculcan a sus hijos una enseñanza enfocada en la importancia de la educación financiera, la compra de activos y el manejo correcto del dinero, mientras que los padres no emprendedores motivan a sus hijos a estudiar fuerte para conseguir trabajos en empresas importantes [18].

- Cultura emprendedora en la universidad: el ambiente emprendedor de la universidad servirá como motivación para los estudiantes que tengan deseos de desarrollar proyectos enfocados en emprendimiento. Algunos de los autores que opinan sobre este factor describen que las universidades emprendedoras tienen el deber de desarrollar una cultura de emprendimiento en toda la comunidad universitaria [19]. Muchos de estos autores reconocen que la cultura emprendedora es fundamental para los estudiantes [14], [16], [20], [21]. Una cultura emprendedora se puede generar por medio de la organización de actividades como talleres y conferencias dictadas por emprendedores, preferiblemente egresados de la institución que está llevado a cabo la actividad, y ferias de emprendimiento, donde los estudiantes puedan exponer sus ideas innovadoras.

- Apoyo institucional: el apoyo que le brinde la institución a los estudiantes juega un papel clave en el desarrollo de innovaciones. Los autores que fueron investigados para este estudio concuerdan en que el rol de la institución para el éxito de los individuos es fundamental [14], [19], [20], [22], [23]. Dentro de las alternativas que pueden ofrecer las universidades para apoyar a sus estudiantes se encuentran la creación de incubadoras de ideas de negocio, la constitución de oficinas de trasferencia de tecnología para el manejo de patentes y propiedad intelectual, convenios que se puedan realizar con otras empresas, la creación de start-ups y spin-offs y el financiamiento de proyectos innovadores.

- Región: el área geográfica donde se desenvuelven las personas es considerado como un factor relevante para muchos autores ya que, la historia emprendedora que acompañe a la región, será influyente en la perspectiva que tengan sus habitantes sobre el emprendimiento. Algunos autores sostienen que, mientras más casos de emprendimiento se desarrollen en una región, se fortalece la cultura emprendedora y los individuos se sentirán motivados a llevar a cabo proyectos innovadores [16], [20]. Dentro de esta investigación se considera un factor influyente, ya que, la cantidad de casos de emprendimiento de éxito, el nivel de urbanización y la economía predominante de la región, determinarán el emprendimiento que desarrollen sus habitantes.

- Edad: es un factor considerado en menor escala por los autores. Dentro de la investigación que se desarrolló, solamente un autor opinó sobre la edad. Este concluyó en su estudio que a mayor edad tenga el estudiante al momento que reciba la educación emprendedora, mayores serán sus intenciones de desarrollar un proyecto innovador [15]. En este estudio, no se considera la edad como un factor relevante, ya que es la madurez, la cual muchas veces es confundida con la edad, la que en verdad influye en las intenciones emprendedores del individuo. La madurez es la que les permite a las personas definir objetivos claros y desarrollar los planes para alcanzarlos.

\subsection{Motivaciones que afectan las intenciones emprendedoras de los individuos}

Además de los factores mencionados en la sección "4.1", es importante tomar en consideración las motivaciones que afectan a las personas, ya que son estas las que logran que los individuos tengan una intención emprendedora y, por consiguiente, desarrollen actividades enfocadas en el emprendimiento. Dentro de esta investigación se consideran como importantes las tres motivaciones más recurrentes en las personas. Estas son las siguientes:

- Necesidad de independencia: característico de aquellos individuos que prefieren asumir riesgos y encuentran satisfacción al conseguir autonomía económica. Se encuentra muy relacionado con los jóvenes de la actualidad que presentan grandes deseos de "ser sus propios jefes" [24], [12].

- Motivación financiera: característico de los individuos que desean mejorar su calidad de vida y ven en el emprendimiento la oportunidad de obtener estabilidad económica. Esta motivación va más allá de lo que el individuo desea para su vida, incluye también a la familia que depende de ellos [12].

- Necesidad de logros: característico de los individuos que disfrutan retarse a sí mismos y, difícilmente, desisten antes de alcanzar sus metas. Estas personas se proponen tareas desafiantes, pero alcanzables. Esta motivación es considerada la más recurrente en los individuos que emprenden. [12], [24] y [25]. 


\subsection{Programas implementados por universidades para incentivar el emprendimiento}

Los mecanismos de enseñanza tradicionales no son óptimos para desarrollar la creatividad en los estudiantes y, por consiguiente, logran disminuir sus deseos emprendedores. Por esto, como se mencionó en la sección "2.3", las universidades se encuentran invirtiendo sus recursos en investigaciones que les permitan actualizar estos métodos. Los programas y modelos que han sido implementados por distintas universidades y son considerados exitosos se presentan en la tabla 2:

Tabla 2. Modelos desarrollados por distintas universidades para incentivar el emprendimiento entre la comunidad estudiantil

\begin{tabular}{|c|c|}
\hline $\begin{array}{l}\text { PROGRAMA O } \\
\text { MODELO }\end{array}$ & BENEFICIO \\
\hline $\begin{array}{l}\text { Modelo de enseñanza } \\
\text { "REPITA" y nuevo } \\
\text { modelo de incubación } \\
\text { de ideas por el } \\
\text { Instituto Tecnológico } \\
\text { de Monterrey [22] }\end{array}$ & $\begin{array}{l}\text { El modelo "REPITA" se enfoca en } \\
\text { distintas áreas que deben ser trabajadas } \\
\text { para desarrollar una cultura de } \\
\text { emprendimiento y el nuevo modelo de } \\
\text { incubación les brinda más opciones de } \\
\text { emprendimiento y desarrollo de } \\
\text { propiedad intelectual a los estudiantes } \\
\text { que el modelo que antes se ofrecía. }\end{array}$ \\
\hline $\begin{array}{l}\text { Política de desarrollo } \\
\text { de emprendimiento y } \\
\text { un programa enfocado } \\
\text { en el desarrollo de } \\
\text { competencias [25] }\end{array}$ & $\begin{array}{l}\text { Este programa ofrece formación en } \\
\text { educación empresarial y educación } \\
\text { emprendedora, donde se hace un } \\
\text { enfoque en desarrollar habilidades } \\
\text { conceptuales, técnicas y blandas. }\end{array}$ \\
\hline $\begin{array}{l}\text { Curso en línea MOOC } \\
\text { [26] }\end{array}$ & $\begin{array}{l}\text { Este programa es recomendado para } \\
\text { aquellas universidades que no poseen } \\
\text { un gran capital para invertir en equipos } \\
\text { novedosos y, a la vez, tienen una gran } \\
\text { demanda de estudiantes. }\end{array}$ \\
\hline $\begin{array}{l}\text { Programa "Formation } \\
\text { Créationd Emploi et de } \\
\text { Travail Indepéndent" } \\
{[17]}\end{array}$ & $\begin{array}{l}\text { Se encarga de desarrollar habilidades } \\
\text { empresariales de administración, de } \\
\text { mercado y blandas. Las ideas, luego de } \\
\text { ser modificadas y perfeccionadas con } \\
\text { la ayuda de un mentor, resultan } \\
\text { óptimas para desarrollar un negocio. }\end{array}$ \\
\hline $\begin{array}{l}\text { Programa para el } \\
\text { desarrollo de estrategias } \\
\text { emprendedoras [27] }\end{array}$ & $\begin{array}{l}\text { Luego de este programa, los } \\
\text { estudiantes presentan una actitud más } \\
\text { estratégica al momento de tomar } \\
\text { decisiones y se encuentran más } \\
\text { enfocados en desarrollo de habilidades } \\
\text { y competencias. }\end{array}$ \\
\hline $\begin{array}{l}\text { Modelo de tres etapas } \\
\text { para estimular al } \\
\text { estudiante emprendedor } \\
{[21]}\end{array}$ & $\begin{array}{l}\text { Este modelo resalta la importancia de } \\
\text { tres puntos clave para una educación } \\
\text { emprendedora exitosa: el desarrollo de } \\
\text { habilidades y espíritu emprendedor, la } \\
\text { enseñanza de habilidades técnicas y el }\end{array}$ \\
\hline
\end{tabular}

\begin{tabular}{|l|l|}
\hline & $\begin{array}{l}\text { apoyo de la institución encargada de la } \\
\text { educación. }\end{array}$ \\
\hline $\begin{array}{l}\text { Modelo } \\
\text { "SCAMPER" [28] }\end{array}$ & $\begin{array}{l}\text { Tiene como finalidad el desarrollo de } \\
\text { la creatividad de los individuos y se } \\
\text { fundamenta en la generación de } \\
\text { nuevas ideas. }\end{array}$ \\
\hline $\begin{array}{l}\text { Maestría de } \\
\text { emprendimiento [29] }\end{array}$ & $\begin{array}{l}\text { Se desarrolla un plan de negocios y se } \\
\text { capacita a los individuos en el ámbito } \\
\text { del emprendimiento y el desarrollo de } \\
\text { habilidades de manejo empresarial. }\end{array}$ \\
\hline
\end{tabular}

\subsection{Modelos propuestos por investigadores para incentivar el emprendimiento}

Además de los modelos presentados anteriormente, muchas investigaciones de profesionales y estudiosos en temas de emprendimiento, se encargan de analizar qué factores deben ser desarrollados y qué estrategias son convenientes para despertar el espíritu emprendedor en los individuos. Algunas de estas propuestas generadas y que con consideradas como relevantes son presentados en la tabla 3:

Tabla 3. Modelos desarrollados por distintos investigadores para incentivar el emprendimiento entre la comunidad estudiantil

\begin{tabular}{|l|l|}
\hline \multicolumn{1}{|c|}{$\begin{array}{c}\text { PROGRAMA O } \\
\text { MODELO }\end{array}$} & \multicolumn{1}{|c|}{ BENEFICIO } \\
\hline $\begin{array}{l}\text { Modelo de distintos } \\
\text { enfoques educativos } \\
\text { [7] }\end{array}$ & $\begin{array}{l}\text { Busca desarrollar en el individuo } \\
\text { habilidades que se complementen con } \\
\text { los conocimientos técnicos específicos } \\
\text { de la carrera profesional que el } \\
\text { individuo estudie o haya estudiado } \\
\text { anteriormente. }\end{array}$ \\
\hline $\begin{array}{l}\text { Programa de enseñanza } \\
\text { emprendedora [30] }\end{array}$ & $\begin{array}{l}\text { Este modelo de enseñanza se } \\
\text { fundamenta en el ensayo y error, donde } \\
\text { se busca perfeccionar un producto o } \\
\text { servicio que pueda ser utilizado como } \\
\text { negocio. }\end{array}$ \\
\hline $\begin{array}{l}\text { Programa de enseñanza } \\
\text { por medio de la } \\
\text { diversificación de ejes } \\
\text { que se desarrollan [8] }\end{array}$ & $\begin{array}{l}\text { Promueve la importancia de la } \\
\text { educación del individuo y del ambiente } \\
\text { institucional óptimo. }\end{array}$ \\
\hline $\begin{array}{l}\text { Enfoques de enseñanza } \\
\text { emprendedora y el } \\
\text { desarrollo de identidad } \\
\text { emprendedora [31] }\end{array}$ & $\begin{array}{l}\text { Argumentan que el mejor enfoque de } \\
\text { enseñanza es el que se genera a través } \\
\text { del emprendimiento y sugieren que la } \\
\text { identidad emprendedora se crea a partir } \\
\text { del desarrollo de habilidades. }\end{array}$ \\
\hline $\begin{array}{l}\text { Modelo de aprendizaje } \\
\text { y apoyo institucional } \\
\text { [23] }\end{array}$ & $\begin{array}{l}\text { Demuestran que la formación de } \\
\text { estudiantes hacia el emprendimiento es } \\
\text { una cadena, donde las decisiones y } \\
\text { apoyo que ofrezca la universidad hacia } \\
\text { los profesores y estudiantes, se verá }\end{array}$ \\
\hline
\end{tabular}




\begin{tabular}{|l|l|}
\hline & $\begin{array}{l}\text { reflejada en el nivel de aprendizaje que } \\
\text { estos últimos alcancen. }\end{array}$ \\
\hline $\begin{array}{l}\text { Modelo de enseñanza } \\
\text { de cuatro etapas [9] }\end{array}$ & $\begin{array}{l}\text { Este modelo de enseñanza tiene como } \\
\text { objetivo el aprendizaje emprendedor y } \\
\text { el aprendizaje empresarial. Además, } \\
\text { señala la importancia dentro de la } \\
\text { educación emprendedora a no buscar } \\
\text { comportamientos similares. }\end{array}$ \\
\hline $\begin{array}{l}\text { Curso de planeamiento } \\
\text { de negocios [10] }\end{array}$ & $\begin{array}{l}\text { El curso tiene como finalidad que los } \\
\text { estudiantes desarrollen confianza a } \\
\text { generar un emprendimiento enfocado } \\
\text { en la rama de estudio que desarrollan. }\end{array}$ \\
\hline
\end{tabular}

\section{Propuesta}

\subsection{Centro de emprendimiento UTP EMPRENDE}

Para desarrollar una propuesta que genere la cultura emprendedora en la comunidad estudiantil de la Universidad Tecnológica de Panamá (UTP) es necesario analizar cuál es el estado existente de la institución. En la actualidad, la universidad cuenta con un centro de emprendimiento llamado "UTP EMPRENDE". El mismo tiene como objetivo cumplir con el compromiso que adquieren las universidades con la sociedad; capacitar de manera integral el capital humano que busca alcanzar conocimiento.

UTP EMPRENDE consta de un equipo capacitado en áreas de administración, economía, proyectos, finanzas, derecho y ciencias políticas, propiedad intelectual, negociación e ingeniería. Este centro se encarga de brindar apoyo, asesoría y seguimiento a proyectos emprendedores dentro y fuera de la Universidad Tecnológica de Panamá, que tienen el potencial para convertirse en negocios exitosos e innovadores [32].

Entre los proyectos que ha desarrollado este centro de emprendimiento se encuentra el Proyecto SIDEP (Sistema de Incubación para el Desarrollo Empresarial de Panamá).

Este tuvo como propósito el desarrollo de un sistema de incubación de proyectos sostenibles para fortalecer y apoyar la creación de nuevas empresas. Otro proyecto desarrollado fue la constitución de cinco nuevos Centros de Emprendimiento en los distintos Centros Regionales de la Universidad Tecnológica de Panamá con la finalidad de llevar el emprendimiento a los distintos sectores del país. Además, se desarrolló un proyecto enfocado en el apoyo a la mujer emprendedora llamado CAME PANAMÁ. Este tuvo como objetivo el asesoramiento en el ámbito empresarial a mujeres con ideas de negocio [32].

El centro de emprendimiento, en este momento, se encuentra enfocado en la incubación de ideas de negocio, pero no cuenta con un modelo de enseñanza para capacitar el capital humano con las habilidades emprendedoras y empresariales necesarias para llevar a cabo un proyecto exitoso. Es por esto, que surge la necesidad de realizar una modificación a los servicios que ofrece el centro de emprendimiento "UTP EMPRENDE", donde se expandan las ofertas educacionales y de formación en beneficio de la comunidad estudiantil de la Universidad Tecnológica de Panamá. La existencia de un centro autónomo e independiente en la universidad es fundamental para que se desarrolle una formación emprendedora de manera correcta. La armonía entre el centro de emprendimiento y la institución de educación superior es clave para el éxito del programa que se presenta [21].

\subsection{Propuesta de modelo para incentivar el emprendimiento de base universitaria}

Para la propuesta de modelo que genere una cultura emprendedora entre los estudiantes de la Universidad Tecnológica de Panamá, se tomaron como base programas y modelos utilizados en distintas universidades, los cuales han sido analizados y se ha demostrado que son exitosos en desarrollar el espíritu emprendedor entre la comunidad estudiantil.

El primero es el curso en línea MOOC (Massive Open Online Course) desarrollado en la Universidad de Taylor en Malasia [26]. El mismo tiene como objetivo principal el desarrollo de habilidades como la descripción del factor clave de una organización, el reconocimiento de las necesidades de los clientes, el manejo efectivo de los recursos, el pensamiento emprendedor, el enfoque emprendedor, la creación de valor, la comunicación emprendedora, el manejo de riesgos empresariales, la construcción de un equipo emprendedor, entre otros [26]. El segundo programa que se utilizará como base es la Maestría en Emprendimiento de la Universidad de Otago en Nueva Zelanda [29]. En este se busca el desarrollo de ejes de aprendizaje como desarrollo de confianza, estudio de factibilidad de ideas, desarrollo de conocimientos y habilidades, realismo ante las oportunidades, identificación de soluciones prácticas, desarrollo de ideas futuras y construcción de redes [29].

Tomando en consideración los modelos mencionados anteriormente, se genera una propuesta para implementar en el centro de emprendimiento "UTP EMPRENDE". Este tiene la finalidad de generar una cultura emprendedora e incentivar el desarrollo de proyectos innovadores en la comunidad estudiantil. Esta propuesta fue dividida en las siguientes siete etapas:

- Primera etapa: se hace un enfoque hacia el aprendizaje de las habilidades conceptuales, técnicas y blandas por medio de capacitaciones. Se introducen conceptos iniciales sobre el emprendimiento como: por qué considerar el emprendimiento como opción para desarrollarse profesionalmente, la identificación de los problemas y las oportunidades que surgen, desarrollo del estilo de pensamiento emprendedor, la construcción de un equipo emprendedor, entre otros. Además de los conceptos básicos, se desarrollan competencias más especializadas y fundamentales para un emprendedor de éxito. Entre las herramientas que se enseñan se tienen: el manejo de proyectos, el manejo de riesgos, la creación de valor para el producto o servicio que se desarrolla, la construcción de estados financieros, el análisis de factibilidad y riesgos, el análisis del valor del dinero en el tiempo, el pago de impuestos, la fijación 
de precios, la distribución de productos, el mercadeo y ventas y desarrollo de estrategias de salida, entre otras.

- Esta primera etapa tiene como objetivo preparar a los individuos para que logren desempeñar de manera óptima sus funciones como propietarios de un negocio. Para esto, de manera general, se desarrollan como temas específicos los conceptos básicos y fundamentales del emprendimiento, el desarrollo del pensamiento emprendedor, la ejecución de funciones de un emprendedor junto con la creación de valor de su producto o servicio, el manejo de riesgos, la proyección de un emprendedor, el manejo de proyectos, la construcción de un equipo emprendedor, el análisis de factibilidad de ideas, el estudio de mercado, las ventas, las finanzas para emprendedores, las estrategias de consolidación de negocios y una especialización en emprendimiento sostenible, emprendimiento enfocado en el turismo o emprendimiento enfocado en la tecnología y la ciencia.

- Segunda etapa: se busca el desarrollo de una idea de negocios factible que sea generada por medio de la creatividad y que sea considerada innovadora. La misma debe tener como objetivo solucionar alguna carencia que haya sido identificada en la sociedad actual. Es preferible que la idea de negocio que desarrolle el individuo se encuentre enfocada en el área profesional en la que se desenvuelve el mismo, de esta manera puede utilizar las habilidades emprendedoras desarrolladas en la primera etapa junto con las habilidades conceptuales y técnicas especializadas de su carrera profesional.

- Tercera etapa: luego de desarrollar una idea de negocio, los individuos deben validar la factibilidad de esta por medio de las herramientas aprendidas en la primera etapa. La validación de las ideas tendrá distintos enfoques, haciendo un balance entre el costo de la implementación y el beneficio que esta ofrece. Es importante tomar en consideración que una idea es considerada factible, no solamente si se puede llevar a cabo y la misma genera ganancias, sino también si se pueden lograr los objetivos esperados al ejecutarla. Entre los análisis que se desarrollan para medir la viabilidad de una idea de negocio se encuentran los análisis de riesgos, los estudios de mercado y los análisis financieros. Estos estudios buscan medir las probabilidades de éxito de la idea de negocios, si se cuenta con la demanda adecuada para que la puesta en marcha de la idea genere ingresos y si el proyecto es factible en términos monetarios, respectivamente.

- Cuarta etapa: luego de validar la viabilidad de la idea, se procede a desarrollar el plan de negocios. Dentro del mismo se incluyen los análisis y estudios aprendidos en la primera etapa. El desarrollo del plan de negocios incluye las generalidades de la empresa y cuál es la visión de la misma, los objetivos que se desean cumplir y los estudios de factibilidad de la idea de negocio. Se resalta la importancia de la construcción adecuada del plan de negocios ya que este servirá para captar el interés de posibles inversores. Este debe ser detallado y a la vez atractivo para las personas que se encuentran fuera del proyecto; debe especificar cuáles son las ventajas competitivas que ofrece la idea, cuáles son las fortalezas que tendrá la empresa, las oportunidades que se presentan en el mercado, definir el sector al cual se le hará la oferta y proyecciones para el futuro.

- Quinta etapa: la incubación de la idea de negocio es fundamental. En esta etapa, el emprendedor o el equipo emprendedor tiene la posibilidad de recibir ayuda personalizada por parte de un mentor especializado en temas de emprendimiento, el cual le ayudará o los ayudará a solventar aquellas dudas que surgen durante la confección del plan de negocios. Además, se encargará de dar seguimiento a los estudios y análisis realizados por medio de sesiones privadas.

- Sexta etapa: la construcción de redes de negocios es el siguiente escalón para pasar de tener una idea de negocio a constituir una empresa. La institución encargada de desarrollar la educación emprendedora, en este caso particular, el centro de emprendimiento "UTP EMPRENDE", adquiere el compromiso de crear redes de negocios para los individuos que participan en el programa de emprendimiento. Las redes de negocios tienen la finalidad de hacer un enlace entre los individuos que desarrollan una idea de negocio, pero no tienen el capital semilla para ponerla en marcha e inversionistas interesados en la idea innovadora.

- Séptima etapa: en esta última etapa del proceso, se busca concluir el ciclo; se tiene como finalidad consolidar la idea de negocio. El centro de emprendimiento debe contar con una oficina de transferencia y su función será fundamental dentro de esta etapa. Para poner en marcha la idea de negocio desarrollada en las etapas anteriores, existen distintas alternativas que se le presentan al emprendedor o equipo emprendedor y estos deben evaluar cuál de las opciones les resulta más conveniente. Entre las opciones que se ofrecen se tienen los "spin-offs", los "startups" y la transferencia de propiedad intelectual.

La propuesta que se realiza tiene como objetivo principal la motivación de los estudiantes hacia el emprendimiento; que los mismos encuentren interés en realizar un proyecto que les apasione $\mathrm{y}$, al mismo tiempo, desarrollen las habilidades que necesitan para llevarlo a cabo.

A continuación, se presenta un diagrama de la propuesta que (figura 1) de manera gráfica de los pasos a seguir y el recorrido del proceso que se presenta: 


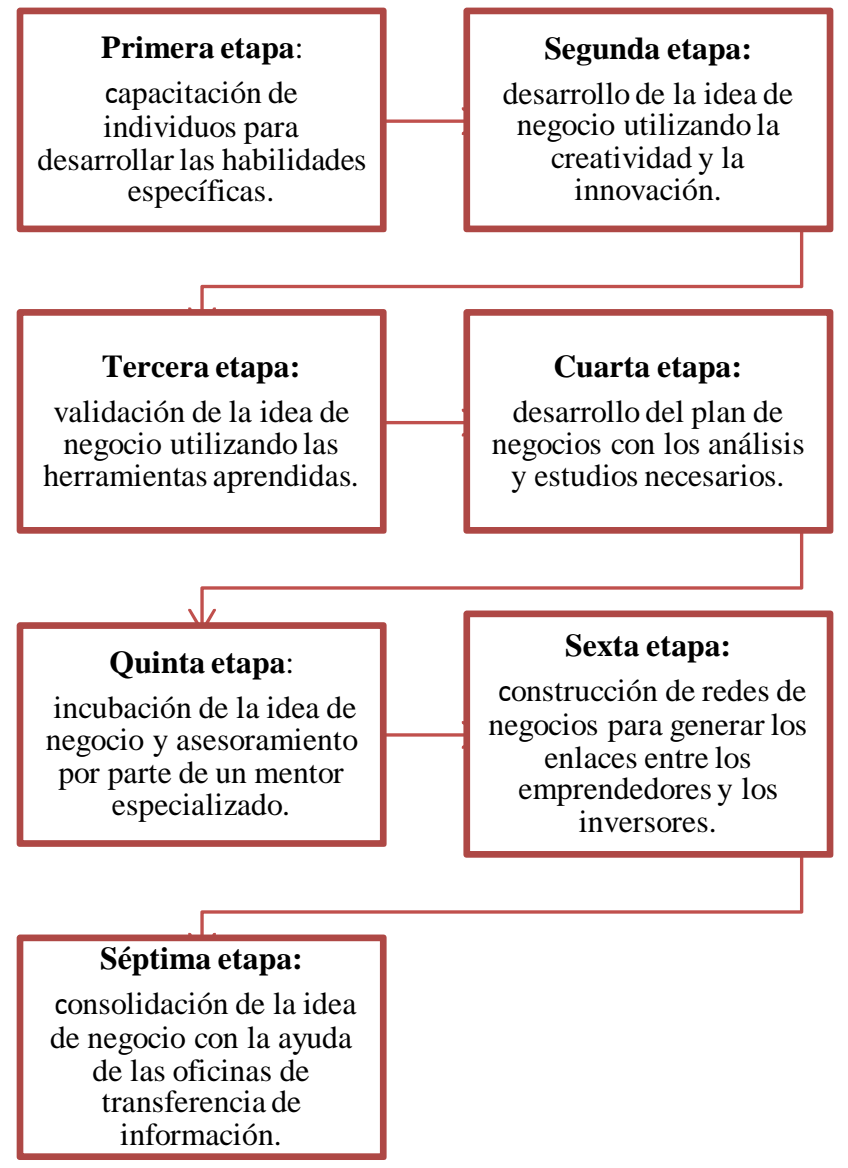

Figura 1: Diagrama de la propuesta para impulsar el emprendimiento en la comunidad estudiantil de la Universidad Tecnológica de Panamá.

\section{Conclusiones}

El emprendimiento se encuentra en un momento de auge, debido a los problemas económicos que presentan las sociedades actuales. Por medio de este estudio, que tuvo como finalidad el desarrollo de un "estado del arte" sobre temas relacionados con emprendimiento de base universitaria en la actualidad, se concluye que se considera este como una solución viable. También, dentro de esta investigación, se confirma que la educación emprendedora tiene efectos positivos en los estudiantes que la reciben.

La institución de educación superior es la encargada de generar una cultura emprendedora entre la comunidad universitaria; esto, complementado con la educación enfocada en el emprendimiento, asegura que los estudiantes desarrollen un espíritu emprendedor que los llevará a la generación de ideas creativas e innovadoras.

La propuesta de modelo para desarrollar e incentivar el emprendimiento entre los estudiantes de la Universidad Tecnológica de Panamá es una composición de distintos programas y cursos que fueron considerados exitosos luego de distintos análisis. El modelo tiene una estructura que se enfoca en el desarrollo de todas las competencias necesarias que convierten a un individuo en un emprendedor de éxito.
Además de esto, el modelo propuesto debe ser complementado con actividades extracurriculares organizadas por el centro de emprendimiento "UTP EMPRENDE". Estas actividades deben enfocarse en el desarrollo del espíritu emprendedor de los estudiantes. Entre las alternativas que se presentan, se consideran efectivas las ferias o competencias emprendedoras, donde los individuos pueden desarrollar una idea innovadora, y las conferencias dictadas por emprendedores de éxito, donde estos comparten sus experiencias.

El modelo de enseñanza emprendedora que se propone tiene la ventaja de que puede ser utilizado por estudiantes que cursen distintas carreras, ya que el mismo desarrolla habilidades generales que pueden ser enfocadas en el emprendimiento independientemente de la rama profesional que siga cada individuo.

Es importante resaltar el papel fundamental que tienen los educadores dentro de la propuesta y el compromiso que posee la institución de educación superior para capacitar a estos, con la finalidad de brindar la mejor formación posible al capital humano que estudia en la Universidad Tecnológica de Panamá.

\section{Recomendaciones}

Luego de la investigación, la propuesta realizada y las conclusiones a las cuales se llegaron se recomienda:

- Modificar los métodos de enseñanza tradicionales para lograr desarrollar en los estudiantes las habilidades y competencias, no solo conceptuales y técnicas, sino también blandas, que son necesarias para que logren afrontar con éxito los retos profesionales que se les presentan y que el individuo pueda desempeñarse de manera óptima en cualquier ámbito en el cual se vea involucrado.

- Invertir en investigaciones, recursos tecnológicos y capacitaciones para los docentes, con el fin de adaptar el sistema educativo y brindar la mejor formación posible a los estudiantes.

- Incentivar el desarrollo de investigaciones futuras por parte de la comunidad estudiantil y docente de la Universidad Tecnológica de Panamá en el ámbito del emprendimiento y enfocadas en el crecimiento y fortalecimiento del centro de emprendimiento que la universidad posee.

Como futura investigación se propone un estudio enfocado en la validación del modelo que se ha propuesto, con la finalidad de analizar cuáles son los efectos que este tiene sobre la población estudiantil. El modelo propuesto se ve abierto a futuras modificaciones dependiendo de los resultados obtenidos.

\section{REFERENCIAS}

[1] R. Cantillon. "Essai Sur la Nature du Commerce en Général." 1755.[En línea]. Disponible: http://biblioteca.libertyfund.org/sites/default/files/naturaleza-delcomercio-electronico.pdf

[2] Banco Mundial. "Nuevas empresas registradas (número)" [En línea].

Disponible: 
https://datos.bancomundial.org/indicador/IC.BUS.NREG?end=20 $16 \&$ name_desc $=$ false $\&$ start $=2010 \& v i e w=$ chart

[3] R. Pérez. "Educación superior en emprendimiento y la superación de la pobreza". Revista Hojas y Hablas, vol. 14, pp. 93-106, 2017.

[4] C. Medina, J. Marin y R. Alfalla. "Una propuesta metodológica para la realización de búsquedas sistemáticas de bibliografía. WPOM, vol. 1(2), pp. 13-30, 2010. Disponible en: https://doi.org/10.4995/wpom.v1i2.786

[5] B. Díaz, J. Gómez, J. García, H. Melo y F. Sanabria. Contribución de las iniciáticas de tecnologías de la información en las organizaciones: una revisión literaria. Innovar, vol. 27(66), pp. 4155, 2017. Disponible en: https://doi.org/10.15446/innovar.v27n66.66710.enlace

[6] D. García y L, Rivera. "estado del arte" sobre el emprendimiento y la creación de empresa. Un enfoque multidisciplinar a partir de Joseph Schumpeter, Edward de Bono, Max Weber, Peters y Waterman”. Universidad de La Salle, Bogotá, 2011.

[7] J. Sánchez, A. Ward, B. Hernández y J. Florez. "Entrepreneurship Education: State of the Art”. Propósitos y Representaciones, vol. 5(2), pp. $401-473, \quad 2017$. Disponible en: http://dx.doi.org/10.20511/pyr2017.v5n2.190

[8] K. Nars y Y. Boujelbene. "Assessing the impact of entrepreneurship education.” ELSEVIER, vol. 109, pp. 712-715, 2014. Disponible en: http://dx.doi.org/10.1016/j.sbspro.2013.12.534

[9] D. Welsh, W. Tullar y H. Nemati. "Entrepreneurship education: Process, method, or both?.” JIK, vol. 1, pp. 125-132, 2016. Disponible en: http://dx.doi.org/10.1016/j.jik.2016.01.005

[10] G. Graevenitz, D. Harhoff y R. Weber. "The effects of entrepreneurship education." ELSEVIER, vol. 76, pp. 90-112, 2010. Disponible en: http://dx.doi.org/10.1016/j.jebo.2010.02.015

[11] T. Jun Bae, S. Quian, C. Miao y J. Fiet. "The Relationship Between Entrepreneuship Education and Entreprenurial Intentions: A Meta-Analytic Review." Wiley Open Library. 2014. Disponible en: http://dx.doi.org/10.1111/etap.12095

[12] V. Barba y C. Atienza. "Entrepreneurial intention among engineering students: The role of entrepreneurship education." European Research on Management and Business Economics, vol. 24, pp. 53-61, 2017. Disponible en: http://dx.doi.org/10.1016/j.iedeen.2017.04.001

[13] E. Novillo, C. Sarmiento, J. Kennedy y D. Ramón. “Análisis de actitud emprendedora en estudiantes universitarios: una perspectiva de género.” INNOVAR, vol. 2, pp. 56-63, 2017.

[14] H. Bergmann, M. Geissler, C. Hundt y B. Grave. "The Climate for Entrepreneurship at Higher Education Institutions." Research Policy, vol. 47, pp. 700-716, 2018. Disponible en: https://doi.org/10.1016/j.respol.2018.01.018

[15] G. Shirokova, O. Osiyevskyy y K. Bogatyreva. "Exploring the intention-behavior link in student entrepreneurship: Moderating effects of individual and environmental characteristics." European Management Journal, vol. 34, pp. 386-399, 2015. Disponible en: http://dx.doi.org/10.1016/j.emj.2015.12.007

[16] M. Obschonka. "The quest for the entrepreneurial culture: psychological Big Data in entrepreneurship research." Current
Opinion in Behavioral Sciences, vol. 18, pp. 69-74, 2017. Disponible en: http://dx.doi.org/10.1016/j.cobeha.2017.07.014

[17] P. Premand, S. Brodmann, R. Almeida, R. Grun y M. Barouni. "Entrepreneurship Education and Entry into Self-Employment Among University Graduates." World Development, vol. 77, pp. 311-327, 2015. Disponible en: http://dx.doi.org/10.1016/j.worlddev.2015.08.028

[18] R. Kiyosaki y S. Lechter. "Padre rico, padre pobre. Qué les enseñan los ricos a sus hijos acerca del dinero, ique las clases media y pobre no!'. Santillana Ediciones Generales S.A. 2004.

[19] N. Yildirim y O. Askun. "Entrepreneurship Intentions of Public Universities in Turkey: Going Beyond Education and Research?" Procedia - Social and Behavioral Sciences, vol. 58, pp. 953-963, 2012. Disponible http://dx.doi.org/10.1016/j.sbspro.2012.09.1074

[20] J. Itri, D. Ballard, S. Kantartzis, J. Sullivan, J. Weisman, D. Durand, S. Ali y A. Kasangra. "Entrepreneurship in the Academic Radiology Environment.” ELSEVIER, vol. 22, pp. 14-24, 2014. Disponible en: http://dx.doi.org/10.1016/j.acra.2014.08.010

[21] S. Jansen, T. Van de Zande, S. Brinkkemper, E. Stam y V. Varma. "How education, stimulation and incubation encourage student entrepreneurship: Observation from MIT, IIIT, and Utrecht University." The International Journal of Management Education, vol. 13, pp. 170-181, 2015. Disponible en: http://dx.doi.org/10.1016/j.ijme.2015.03.001

[22] F. Cantú-Ortíz, N. Galeano, P. Mora-Castro y J. Fangmeyer. "Spreading academic entrepreneurship: Made in Mexico." Business Horizons, vol. 60, pp. 541-550, 2017. Disponible en: http://dx.doi.org/10.1016/j.bushor.2017.04.002

[23] A. Ghina. "Effectiveness of Entrepreneurship Education in Higher Education Institutions." Procedia - Social and Behavioral Sciences, vol. 115, pp. 332-345, 2013. Disponible en: http://dx.doi.org/10.1016/j.sbspro.2014.02.440

[24] J. Sánchez, R. Aldana, S. De Dios y A. Yurrebaso. "La motivación y la intención emprendedora." International Journal of Developmental and Educational Psychology, vol. 4(1), pp. 521531, 2012. Disponible en: http://www.redalyc.org/articulo.oa?id=349832337057

[25] B. $\mathrm{Hj}$, A. Rahim y M. Usman. "The Effectiveness of the Entrepreneurship Education Program in Upgrading Entrepreneurial Skills among Public University Students." Procedia - Social and Behavioral Science, vol. 224, pp, 117-123, $2016 . \quad$ Disponible en: http://dx.doi.org/10.1016/j.sbspro.2016.05.413

[26] M. Al-Atabi y J. DeBoer. "Teaching entrepreneurship using Massive Open Online Course (MOOC)." Technovation, vol. 34, pp. 261-264, 2014. Disponible en: http://dx.doi.org/10.1016/j.technovation.2014.01.006

[27] Y. Stamboulis y A. Barlas. "Entrepreneurship education impact on students attitudes." The International Journal of Management Education, vol. 12, pp. 365-373, 2014. Disponible en: http://dx.doi.org/10.1016/j.ijme.2014.07.001

[28] L. Gundry, L. Ofstein y J. Kickul. "Seeing around corners: How creativity skills in entrepreneurship education influence innovation in business." The International Journal of Management 
Education, vol. 12, pp. 529-538, 2014. Disponible en: http://dx.doi.org/10.1016/j.ijme.2014.03.002

[29] J. Kirkwood, K. Dwyer y B. Gray. "Student's reflections on the value of an entrepreneurship education." The International Journal of Management Education, vol. 12, pp. 307-316, 2014. Disponible en: http://dx.doi.org/10.1016/j.ijme.2014.07.005

[30] K. Oganisjana y T. Laizans. "Opportunity-Oriented ProblemBased Learning for Enhancing Entrepreneurship of University Students." Procedia - Social and Behavioral Sciences, vol. 213, pp. 135-141, 2015. Disponible en: https://doi.org/10.1016/j.sbspro.2015.11.416
[31] A. Donnellon, S. Ollila y K. Williams. "Constructing entrepreneurial identity in entrepreneurship education." The International Journal of Management Education, vol. 12, pp. 490499, 2014.Disponible en: http://dx.doi.org/10.1016/j.ijme.2014.05.004

[32] Universidad Tecnológica de Panamá. “UTP EMPRENDE” [En línea]. Disponible: http://emprende.utp.ac.pa/ 